\title{
Realising Herbert: An Affordable Design Approach of an Anthropometrically Correct Compliant Humanoid Robot
}

\author{
Brennand Pierce and Gordon Cheng \\ Institute for Cognitive Systems, Technische Universität München \\ www.ics.ei.tum.de,Bren@tum.de
}

\begin{abstract}
In this paper we present our new full-sized anthropometrically correct humanoid robot Herbert. Herbert has 18 DOF: 1) 14 active DOFs $(2 \times 1$ DOFs in the knees, $2 \times 3$ in the hips, 3 in the waist and 3 in the head); 2) 4 passive DOFs $(2 \times 2$ in the ankles). We follow a simple design approach while keeping the costs low but ensuring high-performance is achieved. We employed a modular design in realising Herbert. The two core mechanical modules that are used to construct this humanoid robot are: 1) a small compact drive module; and 2) a compliant drive module. We also provide a brief overview of the electronics and the software architecture that support the overall development of this system. Finally, we provide results demonstrating our robot's performances: demonstrating the module's compliant behaviour, the ability of tracking a desired position/velocity as well as a simple torque controller. All in all, we show that our system is compact and able to achieve comparable human performances and proportions.
\end{abstract}

\section{INTRODUCTION}

During the past two decades a number of full sized humanoid robots have been designed and realised: Honda P2/P3[1], HRP-series[2], CB[3], LOLA[4], TORO[5] and ARMAR-4[6], just to name a few. All these humanoid robots are commonly human-sized with a similar performance as a human. All of these humanoids, however, suffer from one common element: their high costs, which are all in the hundreds of thousands of euros (ranging from $€ 200 \mathrm{~K}$ to $€ 1 \mathrm{M}$ ). This is one of the main reasons holding back humanoid robots from leaving the high end laboratories and becoming more widely available in universities, schools and one day into everyday life. The popularity of the NAO humanoid robots [7], which is small and low cost, demonstrates that an inexpensive platform helps to make humanoid research more widely accessible to a larger audience from universities to high schools. Thus, we have been designing a full sized humanoid robot that has similar physical specifications as current full sized humanoid robots, but with the main objective of making it affordable.

1) Key Requirements: Here we outline the key design requirements of Herbert Fig. 1a. It needs to support a wide range of human tasks, therefore, it has been designed to be anthropometrically correct. Hence, the robot has to have the same proportions and weight as a real human [8], as this simplifies direct mapping of human tasks onto the robot, for example in teaching scenarios. It can also then be used in testing prothesis and devices for disabled people or being better able to interact with the human environment around us. This leads to a list of requirements for our new humanoid robot:

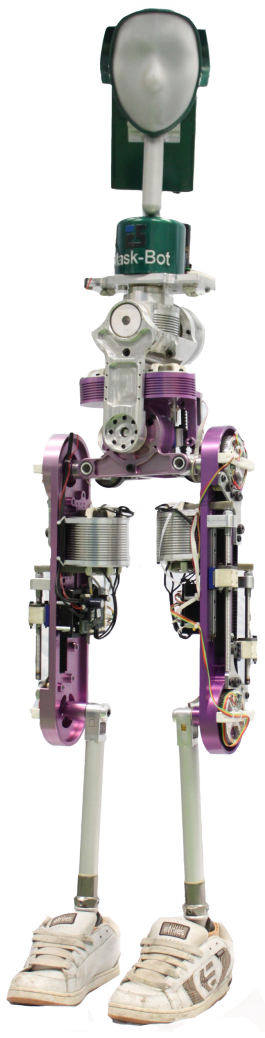

(a)

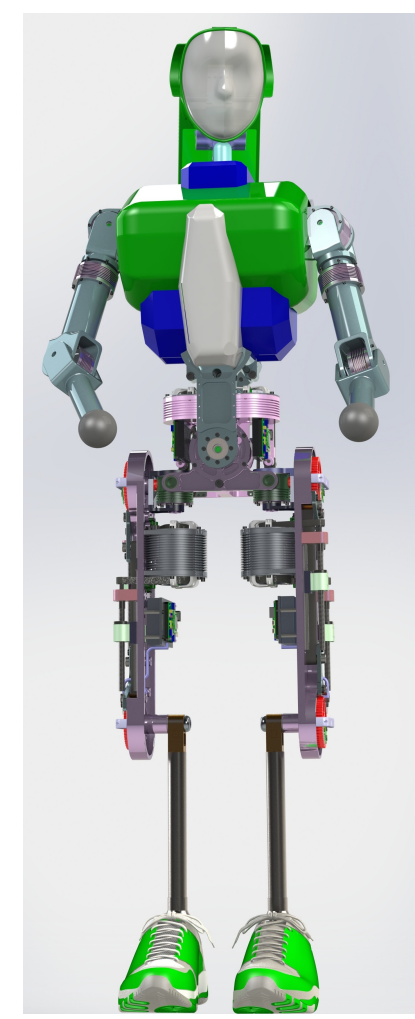

(b)
Fig. 1: Herbert (a) Current state. (b) An CAD render of the completed Herbert including arms.

- Human weight;

- Affordability;

- Compliant joints;

- Anthropometrically correct;

- Torque controllability;

The cost of the actuation mechanism is one of the most significant expenses on any humanoid robot. For example, in hydraulic humanoid robots [3], [9] the high costs are created by the servo valves and the high machining tolerances associated with the pistons; whereas in electric humanoid robots the main costs are the motors, gearboxes and motor controllers. In our approach, we focused on electric actuated humanoid robots as we believe that this approach provides us with the best opportunity of making a full sized humanoid robot at the lowest price while at the same time keeping the same 
physical performance that other robots possess. Looking over the classic design practice of some of the current generation of humanoid robots, we can identify a couple of key areas that can be improved. Firstly, we set out to replace the Harmonic drives [10] that are used in most high performance electric humanoid robots, such as: ARMAR-4[6], iCub[11], TORO[5], HRP-series[2], HUBO[12]. Secondly, we need to examine the power and control electronics followed by the mechanical design with the aim to reduce the costs and simplify the design of the overall system.

Compliant joints have been shown to be beneficial in humanoid robots [13], [14], therefore, we set out to examine the use of compliant joints in a selected number of joints, mainly in the legs. The reason we believe mechanical compliance is important for the humanoid's legs is the foot placement, which is essential for absorbing the impact forces for actions like walking and jumping [15]. Controlled compliance was also considered but that would not provide us with the performance we needed as the latency from the sensors detecting the impact force to the time of the joint moving would be too long [16].

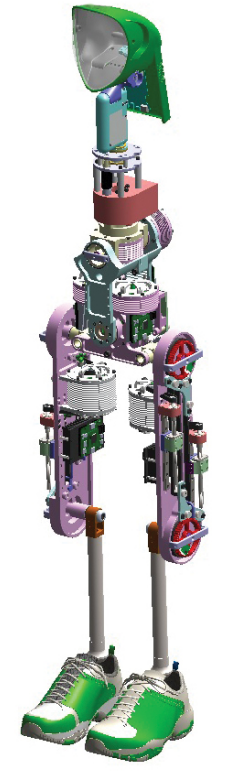

(a)

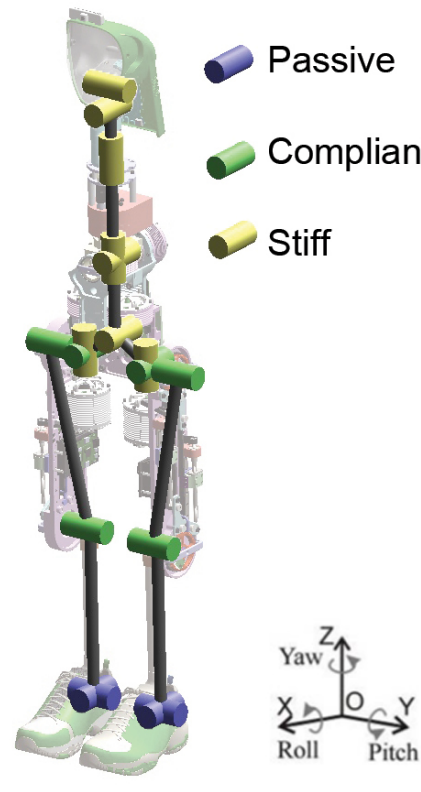

(b)
Fig. 2: Design of Herbert. (a) CAD model. (b) The 18 DOFs. The purple DOFs are the passive prothesis. The green DOFs are the compliant leg joints and the yellow are the low cost stiff joints.

\section{Design And Mechatronics}

Fig. 2a shows the completed computer aided design (CAD) model of Herbert whereas the kinematic structure of the robot is shown in Fig. 2b. As mentioned earlier, our aim for Herbert is to be anthropometrically correct, with the size and weight of a 1 percentile adult male with a hight of 1.59 meters and weight of 45.6 kilograms. The outline of the humanoid and the measurements of the human from [8] are compared

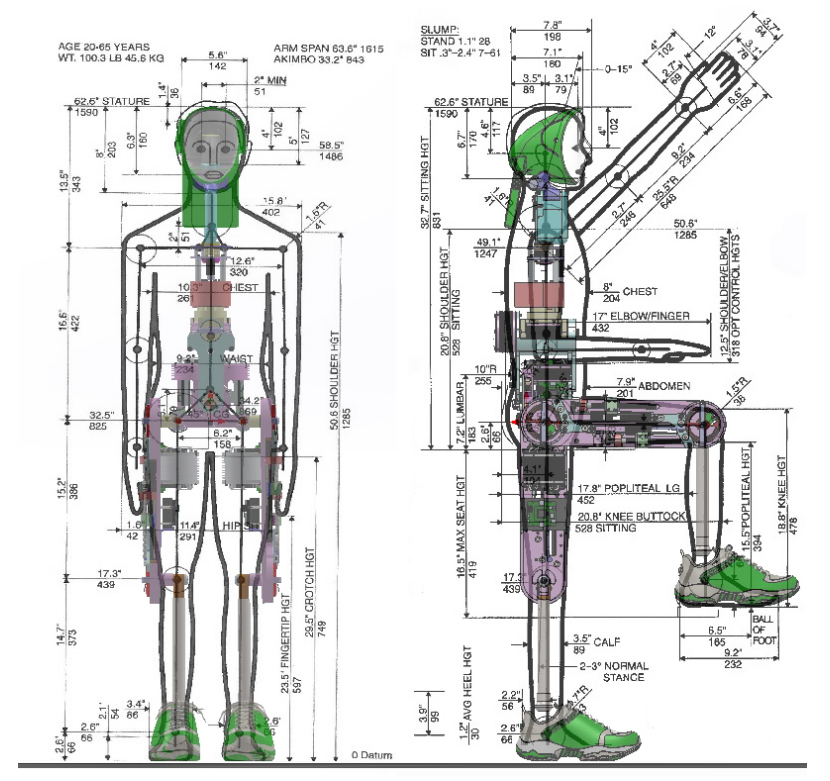

Fig. 3: The human proportion of Herbert: all the major joints are in their anthropometrically correct position, we used the 1 percentile full size human man.

in Fig. 3. Here, the CAD design and the dimensions are displayed super-imposed. It is clear to see in that figure that all the major limbs and joints are in the correct position and the mechanical structures are within the boundaries of the human outline.

For the mechanical structure we designed two types of joint actuation mechanisms. The main joint mechanism is compact and cheap while at the same time being functionally similar to the classical harmonic drive or planetary gearbox design. Most full sized humanoid robots use harmonic drivers for their reduction gearbox as they are small and compact with a high gear ration. Unfortunately, they are very expensive. Planetary gearboxes, on the other hand, have the advantage of low costs but with the drawback of becoming larger and heavier the higher the gear ratio. The second type of joint mechanism in our design is a joint with mechanical compliance. We used the compliant mechanism in the legs, so that it can absorb impacts. For the rest of our humanoid robot we have used the compact design as we believe that the mechanical compliance is not required and therefore software compliance control can be used to save on the overall design complexity and on cost. In table I the types of modules

TABLE I: Torque, range and velocity of the humanoid.

\begin{tabular}{|l|l|c|c|c|c|}
\hline Joint & Type & Ratio & $\begin{array}{c}\text { Torque } \\
{[\mathrm{Nm}]}\end{array}$ & $\begin{array}{c}\text { Range } \\
{[\mathrm{deg}]}\end{array}$ & $\begin{array}{c}\text { Velocity } \\
{[\mathrm{deg} / \mathrm{s}]}\end{array}$ \\
\hline \hline Knee & Compliant & $39.4: 1$ & 99 & $-5,103$ & 457 \\
Hip Pitch & Compliant & $50.3: 1$ & 126 & $-40,87$ & 358 \\
Hip Yaw & Compact & $90: 1$ & 41 & $-19,19$ & 300 \\
Hip Roll & Compliant & $57: 1$ & 141 & $-36,32$ & 573 \\
\hline Waist Roll & Compact & $63: 1$ & 158 & $-20,20$ & 286 \\
Trunk Roll & Compact & $63: 1$ & 158 & $-42,42$ & 286 \\
Trunk Yaw & Compact & $63: 1$ & 158 & $-42,42$ & 286 \\
\hline
\end{tabular}


used in each joint as well as the reduction ratio, maximum torque, joint range and the maximum angularly velocity are presented.

\section{A. Compliant Module}

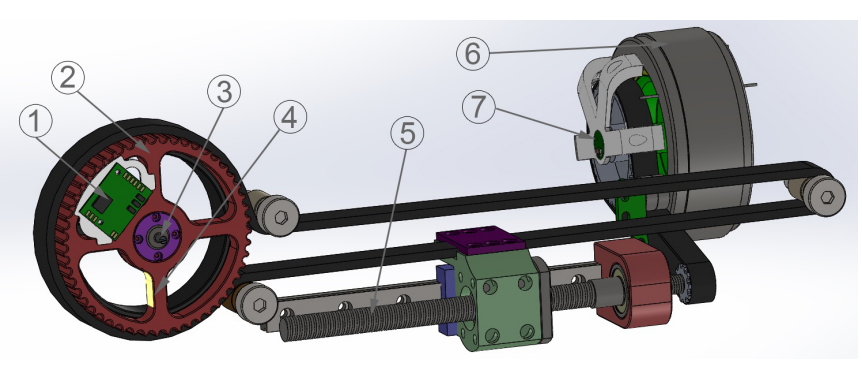

Fig. 4: The compliant joint design.

The hip and knees have been designed with a compliant, back drivable mechanism with a replaceable compliant element. The compliant element can be easily replaced in order to allow experiments into how different complaint behaviours effect the tasks like walking and balancing. As pointed out earlier, it is believed that compliant actuation is very important for walking [15], especially when it comes to heal strike and the inability of precisely predicting the magnitude and time of the impact forces. In Fig. 4 the overview of the compliant joint can be seen. (1) is the digital torque sensor electronics; (2) is the pulley, which is also the "compliant element"; (3) is the digital joint encoder; (4) is the strain gauges; (5) is the ball screw; (6) is the PMSM motor by Robodrive [17]; (7) is the motor position encoder. The design is based around the ball screw that drives the pulley belt. We decided to use a ball screw because it is mechanically back drivable, efficient and low cost. We utilised over sized motors to avoid the need for large gear reductions. With the back drivability and low gear ratio we believe this will give use much better natural dynamics.

The compliant element is directly coupled to the output shaft which has been designed to be easily replaceable. Fig. 5 shows different designs that have been tested in CAD using the finite element method (FEM) to compute the theoretical compliance as well as the strength of the component. By using the FEM analysis we are able to compute the deflection when different torques are applied and also use this data to design the optimum placement for the strain gauges to measure the torque on the real robot. In Fig. 5.2 we experimented with a non-symmetrical design, where one of the spokes was split to increase its length for better placement of stain gauges. We are currently using Fig. 5.3 for Herbert.

This mechanism also has a simple safety feature inherited from the design. This is the ability to measure the joint position and velocity as well as the joint torque with two separate sensors, thus being able to detect sensor failures. To measure the joint position and velocity we primarily use the joint encoder. But we can also use the motor encoder to work out these values, as we know the overall gear reduction. To work out the joint torque we primarily use the strain
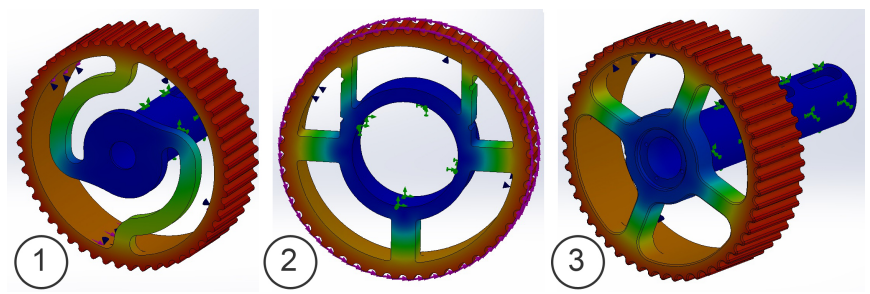

Fig. 5: Example of the FEM analysis for the displacement of the pulley. Herbert is currently using example 3 .

gauges directly mounted at the joint, and also estimate the torque by looking at the difference between the joint position and the motor encoder position. This difference is due to the compliant element as there is a direct correlation between the joint torque and the difference between these two encoders.

\section{B. Compact Module}

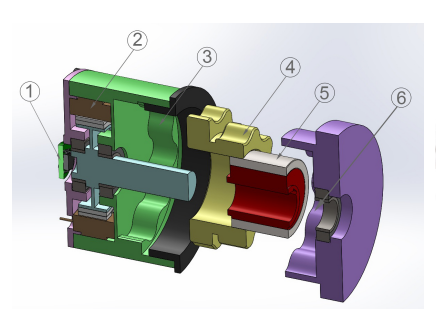

(a)

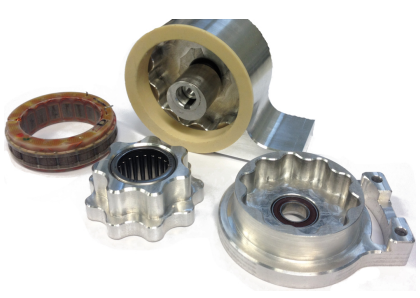

(b)
Fig. 6: Cycloidal reducer. (a) CAD design (b) Example of a real cycloidal reducer.

For the compact design we elected to use a cycloidal reducer [18] instead of the standard method of using harmonic drives. Three advantages are associated with this solution: The first advantage is the cost, as these reducers can be machined at a fraction of the price of a harmonic drive. Secondly, it is compact. Finally, the gear housing and teeth can be machined directly into the main joint structure, which leads into an easier incorporation into the overall design.

The input shaft drives an eccentric bearing that in turn drives the cycloidal disc in an eccentric, cycloidal motion. The perimeter of this disc is geared to a stationary ring gear and as these two connect they make the cycloidal disc rotate at a reduced speed. As we are using a two stage cycloidal reducer this central cycloidal disc then mates to the output shaft. The eccentric motion of the disc is not translated to the output shaft so it is spinning centrically. By varying the number of "teeth" in this design we can obtain a large range of gear ratios for each joint. This works in a similar way to a harmonic drive but with the difference that the harmonic drive has a flexible toothed spline that rotates and the cycloidal reducer has a solid gear centre. The CAD design can be seen in Fig. 6a where (1) is the motor encoder; (2) is the PMSM motor by Robodrive [17]; (3) is the first stage ring gear; (4) is the centre cycloidal disc; (5) is the eccentric input shaft; (6) is the output ring gear. The real version is shown in Fig. 6b. 


\section{Passive Ankles}

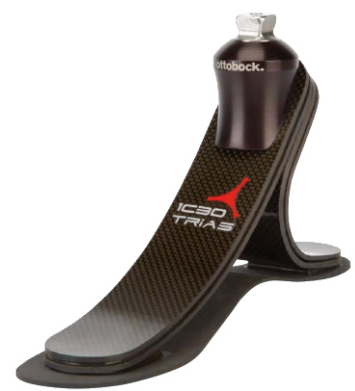

Fig. 7: The prosthetic foot used by Herbert.

To reduce the weight and to simplify the design we have chosen to use human prosthetics for the feet instead of active ankles. This means that the mass of the overall leg is reduced to 5 kilograms and that the centre of mass (CoM) is very high, which has added advantage when it comes to dynamic walking. This also mean that Herbert can be used in the future to help design and verify prosthetic foot designs. With the reduced need for motors, gearboxes and electronics we are also able to reduce the overall cost of the leg design. Herbert currently uses "1C30 Trias" from Otto Bock [19], which can be seen in Fig 7.

\section{Head}

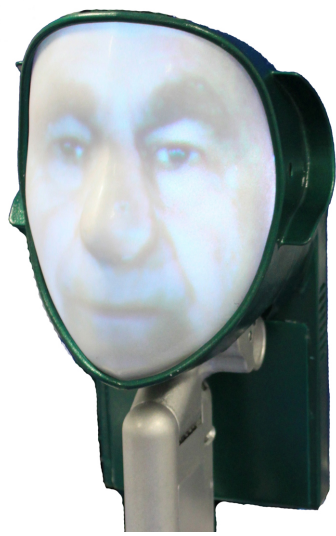

Fig. 8: Head of the humanoid robot [20].

The head of the humanoid robot was developed for human robot interaction and the free standing version is called "Mask-Bot" [20]. This is a rear projected head that is used to display an animated avatar. It is configured with different avatar appearances where eyes and mouth are animated to give a more life-like appearance. The head consists of a rear projector displaying the image onto the face and mounted on a 3 DOF neck. This can be seen in Fig 8 .

\section{ELECTRONICS}

For the electronics of Herbert, we designed our own custom boards based around a modular FPGA design, with each limb having a single board. This modular approach gave us the flexibility to integrate and test different electric
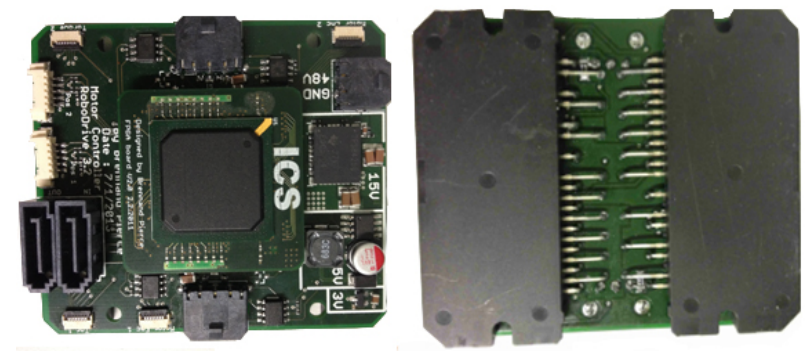

Fig. 9: The electronic board used for the high powered joints

components. The current board used on Herbert is pictured in Fig. 9 with the size of $81 \times 62 \mathrm{~mm}$ and weighing 66 grams. For further details please see our in-depth paper on the electronic system used for this humanoid robot [21]. The communication between the limbs is set at 200Mbps using a custom protocol that works via fibre optic for the inter-board communication. Using fibre optic made the communication immune from the electrical interference coursed by the large electric motors. We used Xsens[22] MTi-30 inertial measurement unit (IMU) in the upper body for precise measurements of the body orientation, as well as embedded IMUs in the FPGA modules in each limb to give good joint position estimation. The motor control boards including the FPGA module is approximately $€ 300$ each, but can drive multiple DOF from a single board.

\section{EXPERIMENTAL RESULTS}

In order to verify our objectives for Herbert we performed a number of experiments, the results of the performance measures will be presented here. Additionally, we also provide an actual detailed weights and costs of the full system in validating our claims.

\section{A. Joint Control and Compliance}

To verify that the compliant actuation design works as expected we performed three experiments. The first two experiments verified that it could track a desired trajectory and torque. The third experiment was to quantify the compliance of the joints.

The first experiment performed a simple PID position controller on the left knee joint. A desired position in the form of a sine wave that slowly increases in amplitude was sent the knee controller. The desired trajectory and the recorded position can be seen in the result shown in Fig. 10. This shows that the knee joint was able to track the desired position with little error and that the knee joint could reach a velocity of $462.8 \mathrm{deg} / \mathrm{s}$.

The second experiment was to validate the torque controller. Again using a simple PID controller we set the desired torque of the hip pitch joint to $0 \mathrm{Nm}$ and pushed the leg with an external force. The results are displayed in Fig. 12. As you can see from the graph an external force was applied pushing the hip up to 90 degrees. Then the external force was removed after 5 second coursing the leg to drop due to gravity. As you can clearly see, the leg swings similar to a dampened pendulum as we haven't compensated for friction 

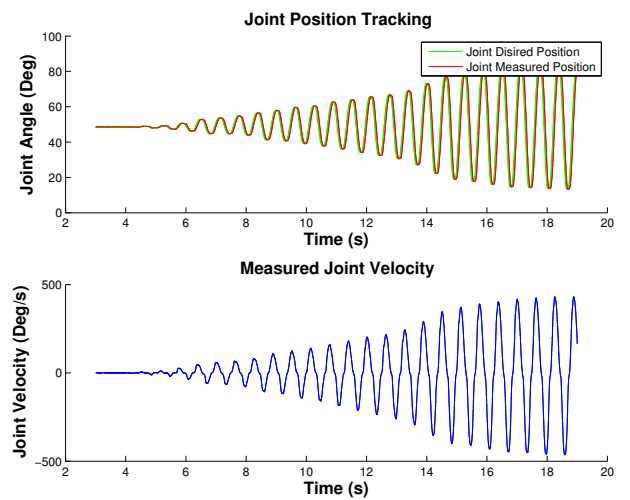

Fig. 10: The top graph shows the joint position tracking a trajectory. The bottom graph shows the measured velocity.

or gravity in the controller. The tracking error is due to the low gains in the controller, with higher gains oscillation is introduced into the system.

The third experiment was to characterise the compliant element of Herbert. We measured an external force which was applied to the hip joint at known distance from the joint axis. We could record this force and the deflexion in the joint angle with the result displayed in Fig.11. As you can see the compliant joint that has a very linear behaviour. The only downside is that at low forces (less than $4 \mathrm{Nm}$ ) there is no compliance. We believe this is due to friction in the system. In table IV you can see the performance of the hip (pitch) and knee joint of Herbert compared to other humanoid robots. It shows that the physical performances of Herbert are well within the range of other available humanoid robots.

\section{B. Weight}

The original target weight for the complete humanoid was 45.6 kilograms, which for the current stage of Herbert would mean that it should weigh about 25 kilograms as we do not have batteries, onboard PC and arms yet. In table II a breakdown of the current weight of our humanoid robot is shown. As you can see from the table the total weight is 21.6 kilograms so Herbert is within the desired weight. As expected, the main weight comes from the lower body. But one really interesting result is the weight of the lower legs as the prosthetic foot saves us a substantial amount of weight. This also means that the upper leg and hip do not need to do

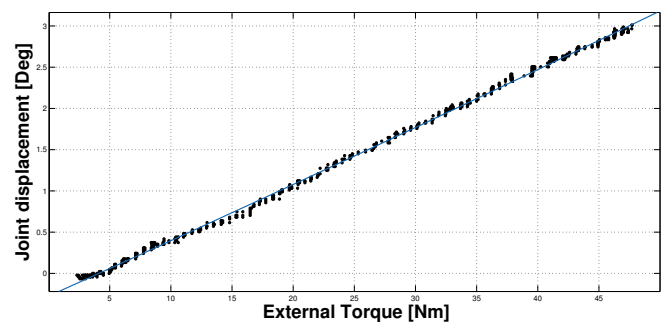

Fig. 11: This shows the displacement of the joint when an external force is applied to it.
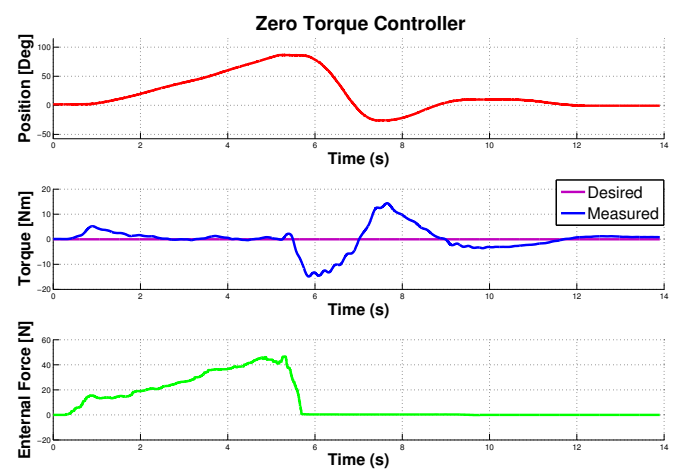

Fig. 12: Experimental result of a simple zero torque loop.

as much work during the swing phase. As shown, the head is very light with most of the weight coming from the projector and the three motors. This is because the majority of the head was 3D printed in plastic which means that we could design a very light shell that acted as the support structure. The centre of mass for this stage of Herbert (including 15 kilograms added to the top for the future weight of the arms) is in the centre of both the $\mathrm{X}$ and $\mathrm{Y}$ plane as the robot was design symmetrically and $14 \mathrm{~mm}$ below the waist roll joint in the $\mathrm{Z}$ plane, which is very similar to a human.

\section{DISCUSSION}

One of the primary aims for this humanoid robot was lowering the cost compared to current humanoids available. In table III we compare the estimated cost of our completed humanoid (including arms and batteries) with other humanoids. This table therefore shows that in the future we could be able to produce a complete humanoid robot for under $€ 40,000$. Although, this sum excludes the labour but it is including the estimated cost of two complete arms, batteries and onboard computers. A projection of how this finished humanoid could look like is shown in Fig.1b. It is obvious that the main expenses are the motors as the Robodrives are roughly $€ 800$ each. Nevertheless, it is true that the costs of the mechanical components, which include the cost of the ball screw and cycloidal reducer, are very low as a lot of effort was put into reducing the number of components used and the cost of manufacturing these components. Over a couple of iterations we were also able to combine different structural components

TABLE II: Weight of the Hu- TABLE III: Cost of the Humanoid.

\begin{tabular}{|l|r|}
\hline Part & Weight $[\mathrm{Kg}]$ \\
\hline Upper Leg & 4.32 \\
Lower Leg + Foot & 0.65 \\
Waist & 5.11 \\
\hline Lower Subtotal & $\mathbf{1 5 . 0 5}$ \\
\hline Lower Waist & 2.19 \\
Upper Body & 2.89 \\
Head & 2.4 \\
\hline Upper Subtotal & $\mathbf{7 . 4 8}$ \\
\hline Total & $\mathbf{2 1 . 6} \mathbf{~ k g}$ \\
\hline
\end{tabular}
manoid.

\begin{tabular}{|l|r|}
\hline Part & Cost in $€$ \\
\hline Motors & 9,569 \\
Encoders & 655 \\
Electronics & 3,080 \\
Prothesis & 2,236 \\
Mechanical & 5,481 \\
\hline Total & $€ \mathbf{2 1 , 0 2 2}$ \\
\hline
\end{tabular}


TABLE IV: Physical Performance and Price Comparison.

\begin{tabular}{|c|c|c|c|c|c|c|c|c|c|c|}
\hline Type & Unit & Herbert & Hubo & Wabian-2 [23] & Sarcos [3] & ETL [24] & Toro [5] & Lola [4] & Armar 4 [6] & Human [8] \\
\hline \multicolumn{11}{|c|}{ Knee Joint } \\
\hline Torque & {$[\mathrm{Nm}]$} & 99 & 72 & 150.9 & 300 & 84 & 180 & 120 & 157 & 71.4 \\
\hline Range & {$[\mathrm{Deg}]$} & $-103,5$ & - & - & - & $-141,5$ & $-115,115$ & $-125,5$ & $-110,0$ & $-122,0$ \\
\hline Velocity & {$[\mathrm{Deg} / \mathrm{s}]$} & 457 & 500 & 314 & 450 & 315 & 110 & 689 & 336 & 446 \\
\hline Gear Ratio & & $39.4: 1$ & $120: 1$ & $365: 1$ & $\mathrm{n} / \mathrm{a}$ & 100:1 & $160: 1$ & $55: 1$ & 100:1 & $\mathrm{n} / \mathrm{a}$ \\
\hline \multicolumn{11}{|c|}{ Hip Pitch Joint } \\
\hline Torque & {$[\mathrm{Nm}]$} & 126 & 60.2 & 55.8 & 150 & 84 & 100 & 125 & 157 & 111 \\
\hline Range & [Deg] & $-40,87$ & - & - & - & $-20,120$ & $-60,120$ & $-45,110$ & $-25,70$ & $-45,140$ \\
\hline Velocity & {$[\mathrm{Deg} / \mathrm{s}]$} & 358 & 300 & 386 & 450 & 315 & 120 & 690 & 336 & 206 \\
\hline Gear Ratio & & 50.3:1 & $200: 1$ & 181:1 & $\mathrm{n} / \mathrm{a}$ & 100:1 & 160:1 & $50: 1$ & 100:1 & $\mathrm{n} / \mathrm{a}$ \\
\hline \multicolumn{11}{|c|}{ Estimated Cost } \\
\hline & Euros & $\sim € 40 \mathrm{k}$ & $\sim € 200 \mathrm{~K}$ & $\sim € 300 \mathrm{~K}$ & $\sim € 1 \mathrm{M}$ & $\sim € 1 \mathrm{M}$ & $\sim € 400 \mathrm{k}$ & $\sim € 300 \mathrm{k}$ & $\sim € 300 \mathrm{k}$ & $\mathrm{n} / \mathrm{a}$ \\
\hline
\end{tabular}

into a single part. Although it was more complex to machine this single part, it was overall cheaper than the combined cost of a number of smaller and more simplified parts. Also 90 percent of the parts were manufactured in-house with low-cost CNC machines which shows our design has been optimised for simplified production. In table IV you can see a comparison between Herbert's and other high performance humanoids' price. As shown, Herbert is substantially cheaper than currently available humanoid robots with similar physical performance. We have also estimated the complete weight of the finished robot to be $\sim 41$ kilograms in total.

\section{CONCLUSION}

In this paper we have shown that using a mixture of cycloidal reducers and our compliant joint design makes it possible to reduce the cost of a humanoid robot while keeping the same high physical performance as other humanoid robots. Furthermore, Herbert is anthropometrically correct and has a weight of 21.6 kilograms and costs $€ 21,022$ (excluding batteries, arms and embedded computer).

In the future, we wish to complete Herbert with a pair of $7 \mathrm{DOF}$ arms which uses a combination of the cycloidal reducer and compliant joint design that we showed in this paper. The projection can be seen in Fig. $1 \mathrm{~b}$ and a final cost should be $\sim € 40,000$ and total weight of $\sim 41$ kilograms.

\section{ACKNOWLEDGMENT}

This work was supported by the DFG cluster of excellence 'Cognition for Technical Systems - CoTeSys' of Germany.

\section{REFERENCES}

[1] K. Hirai, M. Hirose, Y. Haikawa, and T. Takenaka, "The Development of Honda Humanoid Robot," in Proceedings of the 1998 IEEE International Conference on Robotics and Automation, Leuven, Belgium, May 1998, pp. 1321-1326.

[2] K. Kaneko, K. Harada, F. Kanehiro, G. Miyamori, and K. Akachi, "Humanoid robot HRP-3," in 2008 IEEE/RSJ International Conference on Intelligent Robots and Systems. IEEE, Sep. 2008, pp. 2471-2478.

[3] G. Cheng, S. Hyon, J. Morimoto, A. Ude, J. G. Hale, G. Colvin, W. Scroggin, and S. C. Jacobsen, "CB: A Humanoid Research Platform for Exploring NeuroScience," Advanced Robotics, vol. 21, no. 10, pp. 1097-1114, May 2007.

[4] S. Lohmeier, T. Buschmann, and H. Ulbrich, "System Design and Control of Anthropomorphic Walking Robot LOLA," Mechatronics, IEEE/ASME Transactions on, vol. 14, no. 6, pp. 658-666, 2009.

[5] C. Ott, C. Baumg, J. Mayr, M. Fuchs, R. Burger, D. Lee, O. Eiberger, A. Albu-sch, M. Grebenstein, and G. Hirzinger, "Development of a Biped Robot with Torque Controlled Joints," in International Conference on Humanoid Robots, 2010, pp. 167-173.
[6] T. Asfour, J. Schill, H. Peters, J. B. C. Klas, C. Sander, S. Schulz, A. Kargov, T. Werner, and V. Bartenbach, "Armar-4: A 63 dof torque controlled humanoid robot," in IEEE/RAS International Conference on Humanoid Robots (Humanoids), 2013, pp. 0-0.

[7] D. Gouaillier, V. Hugel, P. Blazevic, C. Kilner, P. Lafourcade, B. Marnier, J. Serre, and B. Maisonnier, "Mechatronic design of NAO humanoid," in Conference on Robotics and Automation, 2009, pp. $769-774$.

[8] A. R. Tilley, The Measure of Man and Woman: Human Factors in Design, H. D. Associates, Ed. John Wiley and Sons, Inc., 2002, ISBN: 0-471-09955-4.

[9] S. Alfayad, F. B. Ouezdou, and F. Namoun, "New 3DOF Hybrid Mechanism for Ankle, Wrist of Humanoid Robot : Modeling, Simulation, Control and Experiments," in Journal of Mechanical Design, vol. 133, no. 2, Feb. 2011.

[10] Harmonic Drive AG. [Online]. Available: http://www.harmonicdrive. de

[11] A. Parmiggiani, G. Metta, and N. Tsagarakis, "The mechatronic design of the new legs of the iCub robot," in IEEE-RAS International Conference on Humanoid Robots, 2012, pp. 481-486.

[12] J.-h. Oh, D. Hanson, W.-s. Kim, Y. Han, J.-y. Kim, and I.-w. Park, "Design of Android type Humanoid Robot Albert HUBO," in 2006 IEEE/RSJ International Conference on Intelligent Robots and Systems. IEEE, Oct. 2006, pp. 1428-1433.

[13] A. Enoch, A. Sutas, S. Nakaoka, and S. Vijayakumar, "BLUE : A Bipedal Robot with Variable Stiffness and Damping," in IEEE-RAS International Conference on Humanoid Robots, 2012, pp. 487-494.

[14] N. G. Tsagarakis, J. Saglia, and D. G. Caldwell, "The design of the lower body of the compliant humanoid robot "cCub"," in 2011 IEEE International Conference on Robotics and Automation. IEEE, May 2011, pp. 2035-2040.

[15] J. W. Hurst, "The electric cable differential leg: a novel design approach for walking and running," International Journal of Humanoid Robotics, vol. 8, no. 02, pp. 301-321, 2011.

[16] G. A. Pratt and M. M. Williamson, "Series elastic actuators," in Intelligent Robots and Systems 95.'Human Robot Interaction and Cooperative Robots', Proceedings. 1995 IEEE/RSJ International Conference on, vol. 1. IEEE, 1995, pp. 399-406.

[17] RoboDrive GmbH. [Online]. Available: http://www.robodrive.de

[18] Cycloidal Drive. [Online]. Available: http://www.wikipedia.org/wiki/ cycloidal $\backslash$ drive

[19] Otto bock Plc. [Online]. Available: http://www.ottobock.com

[20] B. Pierce, K. Takaaki, V. Christian, and G. Cheng, "“Mask-Bot 2i": An active customisable Robotic Head with Interchangeable Face," in Proceedings of IEEE-RAS International Conference on Humanoid Robots (Humanoids 2012), 2012, pp. 520-525.

[21] B. Pierce and G. Cheng, "Versatile Modular Electronics for Rapid Design and Development of Humanoid Robotic Subsystems," in Proceedings of IEEE/ASME International Conference on Advanced Intelligent Mechatronics (AIM 2014), 2014.

[22] Xsens Technologies. [Online]. Available: http://www.xsens.com

[23] H. Aikawa, K. Shimomura, H. Kondo, A. Morishima, and A. Takanishi, "Development of a new humanoid robot WABIAN-2," in Proceedings 2006 IEEE International Conference on Robotics and Automation, 2006. ICRA 2006. IEEE, May 2006, pp. 76-81.

[24] G. Cheng, "A High-Performance Full Body Humanoid System For versatile Real world Interaction," in The ELT-Humanoid system, 2003. 